\title{
Root separation for reducible integer polynomials
}

\author{
Yann Bugeaud and Andrej Dujella
}

\begin{abstract}
We construct parametric families of (monic) reducible polynomials having two roots very close to each other.
\end{abstract}

\section{Introduction}

The (naïve) height $H(P)$ of an integer polynomial $P(x)$ is the maximum of the absolute values of its coefficients. For a separable integer polynomial $P(x)$ of degree $d \geq 2$ and with distinct roots $\alpha_{1}, \ldots, \alpha_{d}$, we set

$$
\operatorname{sep}(P)=\min _{1 \leq i<j \leq d}\left|\alpha_{i}-\alpha_{j}\right|
$$

and define the quantity $e(P)$ by

$$
\operatorname{sep}(P)=H(P)^{-e(P)} .
$$

Following the notation introduced in [7], for $d \geq 2$, we set

$$
e(d):=\limsup _{\operatorname{deg}(P)=d, H(P) \rightarrow+\infty} e(P)
$$

and

$$
e_{\mathrm{irr}}(d):=\limsup _{\operatorname{deg}(P)=d, H(P) \rightarrow+\infty} e(P),
$$

where the latter limsup is taken over the irreducible integer polynomials $P(x)$ of degree $d$. We further define $e^{*}(d)$ and $e_{\text {irr }}^{*}(d)$ by restricting to monic,

${ }^{0} 2010$ Mathematics Subject Classification 11C08, 11J04. Keywords: Polynomial, root separation.

The authors were supported by the French-Croatian bilateral COGITO project Polynomial root separation. 
respectively, monic irreducible integer polynomials, of degree $d$. Obviously, we have

$$
e(d) \geq e_{\mathrm{irr}}(d) \quad \text { and } \quad e^{*}(d) \geq e_{\mathrm{irr}}^{*}(d), \quad(d \geq 2) .
$$

A classical result of Mahler [11] asserts that $e(d) \leq d-1$ for every $d \geq 2$ and it is easy to check that $e_{\text {irr }}(2)=e(2)=1$ and $e^{*}(2)=e_{\text {irr }}^{*}(2)=0$.

The determination of the exact values of $e(d), e^{*}(d), e_{\mathrm{irr}}(d)$ and $e_{\mathrm{irr}}^{*}(d)$ has been investigated by several authors over the past ten years. Evertse [10] and Schönhage [12] proved, independently, that $e_{\text {irr }}(3)=e(3)=2$. Rather surprisingly, no other known value of $e(d)$ is known. The following inequalities gather the lower bounds obtained by Beresnevich, Bernik, Bugeaud, Dujella, Götze, and Mignotte in the four papers [6, 7, 1, 5]:

$$
\begin{gathered}
e_{\mathrm{irr}}(d) \geq \frac{d}{2}+\frac{d-2}{4(d-1)}, \quad \text { for } d \geq 4, \\
e(d) \geq \frac{d+1}{2}, \quad \text { for odd } d \geq 5, \\
e^{*}(3)=e_{\mathrm{irr}}^{*}(3) \geq \frac{3}{2}, \quad e^{*}(5) \geq 2, \quad e_{\mathrm{irr}}^{*}(5) \geq \frac{7}{4}, \\
e^{*}(d) \geq \frac{d}{2}, \quad e_{\mathrm{irr}}^{*}(d) \geq \frac{d-1}{2}, \quad \text { for even } d \geq 4,
\end{gathered}
$$

and

$$
e^{*}(d) \geq \frac{d-1}{2}, \quad e_{\mathrm{irr}}^{*}(d) \geq \frac{d}{2}+\frac{d-2}{4(d-1)}-1, \quad \text { for odd } d \geq 7 .
$$

See also [9] for a constructive proof that $e^{*}(4) \geq 2$ and [8] for the study of analogous quantities defined in terms of the Remak height (instead of the naïve height).

The aim of the present paper is to improve all known lower bounds for $e(d), e^{*}(d)$ and $e_{\mathrm{irr}}^{*}(d)$ for $d$ sufficiently large.

Theorem 1 For any integer $d \geq 4$, we have

$$
e(d) \geq \frac{2 d-1}{3}
$$

We obtain a slightly weaker lower bound when we restrict our attention to monic polynomials. 
Theorem 2 For any even positive integer $d \geq 6$, we have

$$
e^{*}(d) \geq \frac{2 d-3}{3} .
$$

For any odd positive integer $d \geq 7$, we have

$$
e^{*}(d) \geq \frac{2 d-5}{3} .
$$

Roughly speaking, all the previously known lower bounds were of order $d / 2$. There are many other questions on integer polynomials of degree $d$, or on algebraic numbers of degree $d$, for which the answer is known to lie somewhere between $d / 2$ and $d$. The most celebrated one is the problem of Wirsing on the approximation to transcendental real numbers by algebraic numbers of degree at most $d$; see Chapter 3 of [4]. We stress that the lower bounds in Theorems 1 and 2 are of the order $2 d / 3$. As far as we are aware, this is the first time where an estimate of order $\theta d$ with $\theta>1 / 2$ is obtained for such kind of questions.

As pointed out in [3], irreducible polynomials with close roots are useful to investigate the difference between Mahler's and Koksma's classifications of real numbers. However, it does not seem to us that Theorem 1 could be applied to this question to improve Corollary 1 of [5].

To prove Theorems 1 and 2 we construct parametric families of integer polynomials. For an integer $d \geq 4$, the reducible polynomials arising in the proof of Theorem 1 are products of a linear polynomial $L_{n}(x)=\left(n^{2}+\right.$ $3 n+1) x-(n+2)$ with an irreducible polynomial $p_{d-1, n}(x)$ of degree $d-1$ and height of order $n$. We then show that $p_{d-1, n}(x)$ has a root $y_{d, n}$ very close to the root $x_{n}=(n+2) /\left(n^{2}+3 n+1\right)$ of $L_{n}(x)$. Say differently, we construct a parametric family $\left(y_{d, n}\right)_{n \geq 1}$ of algebraic numbers of degree $d-1$ which are very well approximated by a rational number with large height (in comparison to the height of $y_{d, n}$ ). This means that it is then possible to apply Bombieri's version of the Thue-Siegel principle [2, Theorem 4] to the anchor pair $\left(y_{d, n}, x_{n}\right)$ to derive a rather good effective irrationality measure for $y_{d, n}$ when $n$ is sufficiently large in comparison to $d$. In his paper, Bombieri used the polynomials $x^{d}-n x+1$, which have a root very close to the rational number $1 / n$.

In our last result, we improve the known lower bound for $e_{\mathrm{irr}}^{*}(d)$ when $d$ is large enough.

Theorem 3 For any positive integer $d \geq 4$, we have

$$
e_{\mathrm{irr}}^{*}(d) \geq \frac{d}{2}-\frac{1}{4} .
$$


Throughout the next sections, the constants implied by the symbols $O$, $\ll$ and $\gg$ can be explicitly computed, are independent of the parameter $n$, and depend at most on the degree $d$.

\section{Reducible polynomials: Proof of Theorem 1}

We want to construct a one-parametric sequence of integer polynomials $p_{d, n}(x)$ of degree $d$ having a root very close to the rational number $x_{n}=$ $(n+2) /\left(n^{2}+3 n+1\right)$. Then the polynomials

$$
P_{d, n}(x)=\left(\left(n^{2}+3 n+1\right) x-(n+2)\right) p_{d-1, n}(x)
$$

will have two roots very close to each other. We define the sequence $p_{d, n}(x)$ recursively by

$$
\begin{aligned}
& p_{0, n}(x)=-1, \quad p_{1, n}(x)=(n+1) x-1, \\
& p_{d, n}(x)=(1+x) p_{d-1, n}(x)+x^{2} p_{d-2, n}(x) .
\end{aligned}
$$

We claim that

$$
p_{d, n}\left(\frac{n+2}{n^{2}+3 n+1}\right)=\frac{(-1)^{d-1}}{\left(n^{2}+3 n+1\right)^{d}} .
$$

Indeed, (2) is clearly true for $d=0$ and $d=1$. Assume now that $d \geq 1$ is an integer for which (2) holds for $p_{d-1, n}(x)$ and $p_{d, n}(x)$. Then we deduce from the recursion (1) that

$$
\begin{aligned}
p_{d, n}\left(\frac{n+2}{n^{2}+3 n+1}\right)= & \frac{(-1)^{d-2}}{\left(n^{2}+3 n+1\right)^{d-1}} \cdot \frac{n^{2}+4 n+3}{n^{2}+3 n+1} \\
& +\frac{(-1)^{d-3}}{\left(n^{2}+3 n+1\right)^{d-2}} \cdot \frac{n^{2}+4 n+4}{\left(n^{2}+3 n+1\right)^{2}} \\
= & \frac{(-1)^{d-1}}{\left(n^{2}+3 n+1\right)^{d}},
\end{aligned}
$$

as claimed.

We now show that for sufficiently large $n$ the polynomial $p_{d, n}(x)$ has a root between $x_{n}$ and

$$
z_{d, n}=x_{n}+\frac{(-1)^{d}}{n\left(n^{2}+3 n+1\right)^{d}} .
$$


Observe that

$$
(-1)^{d-1} p_{d, n}\left(x_{n}\right)=\frac{1}{\left(n^{2}+3 n+1\right)^{d}}>0 .
$$

By Rolle's theorem, there exists $z_{d, n}^{\prime}$ between $x_{n}$ and $z_{d, n}$ such that

$$
p_{d, n}\left(z_{d, n}\right)=p_{d, n}\left(x_{n}\right)+\left(z_{d, n}-x_{n}\right) p_{d, n}^{\prime}\left(z_{d, n}^{\prime}\right) .
$$

It follows easily by induction that

$$
p_{d, n}(x)=-1+(n-d+2) x+((d-1) n-(d-1)(d-2) / 2) x^{2}+\cdots
$$

Since $x_{n}=1 / n+O\left(1 / n^{2}\right)$, we have $p_{d, n}^{\prime}\left(z_{d, n}^{\prime}\right)=n+d+O(1 / n)$. Thus, for sufficiently large $n$, we get $p_{d, n}^{\prime}\left(z_{d, n}^{\prime}\right)>n$. This implies that

$$
(-1)^{d-1} p_{d, n}\left(z_{d, n}\right)=\frac{1}{\left(n^{2}+3 n+1\right)^{d}}-\frac{1}{n\left(n^{2}+3 n+1\right)^{d}} p_{d, n}^{\prime}\left(z_{d, n}^{\prime}\right)<0 .
$$

Therefore, the polynomial $P_{d, n}(x)=\left(\left(n^{2}+3 n+1\right) x-(n+2)\right) p_{d-1, n}(x)$ has two close roots: $x_{n}$ and $y_{d, n}$, which is between $x_{n}$ and $z_{d-1, n}$. This yields

$$
\operatorname{sep}\left(P_{d, n}\right) \leq\left|x_{n}-y_{d, n}\right| \leq \frac{1}{n\left(n^{2}+3 n+1\right)^{d-1}} \leq \frac{1}{n^{2 d-1}},
$$

when $n$ is large enough. Since the height of $P_{d, n}(x)$ is bounded from above by $n^{3}$ times a number depending only on $d$, this gives

$$
e(d) \geq \frac{2 d-1}{3}
$$

by letting $n$ tend to infinity. The proof of Theorem 1 is complete.

By Gelfond's inequality (see e.g. [4, Lemma A.3]), the height of $y_{d, n}$ is $\ll n$. Liouville's inequality (see e.g. [4, Theorem A.1]) then implies that

$$
\left|x_{n}-y_{d, n}\right| \gg n^{-2 \delta-1},
$$

where $\delta$ denotes the degree of $y_{d, n}$. Combined with (3), this gives $\delta=d-1$ and establishes that the polynomial $p_{d-1, n}(x)$ must be irreducible. Also, (3) shows that Liouville's inequality

$$
\left|x_{n}-y_{d, n}\right| \gg n^{-2 d+1}
$$

is sharp in terms of $n$. 


\section{Reducible monic polynomials: Proof of Theo- rem 2}

In order to get a family of monic polynomials with similar separation properties as the family $P_{d, n}(x)$, we replace the linear non-monic polynomial $L_{n}(x)=\left(n^{2}+3 n+1\right) x-(n+2)$ by the monic quadratic polynomial

$$
K_{n}(x)=x^{2}-\left(n^{2}+3 n+1\right) x+(n+2) .
$$

Thus, we want to construct a one-parametric sequence of integer polynomials $q_{d, n}(x)$ of degree $d$ having a root very close to the root $y_{n}=1 / n+O\left(1 / n^{2}\right)$ of $K_{n}(x)$. Then the polynomials

$$
Q_{d, n}(x)=\left(x^{2}-\left(n^{2}+3 n+1\right) x+(n+2)\right) q_{d-2, n}(x)
$$

will have two roots very close to each other.

For $d \geq 0$ even, we define the sequence $q_{d, n}(x)$ recursively by

$$
\begin{aligned}
& q_{0, n}(x)=1, \quad q_{2, n}(x)=x^{2}-(n+1) x+1, \\
& q_{d, n}(x)=\left(2 x^{2}+x+1\right) q_{d-2, n}(x)-x^{4} q_{d-4, n}(x) .
\end{aligned}
$$

We claim that $q_{d, n}(x)-q_{d-2, n}(x) q_{2, n}(x)$ is divisible by $K_{n}(x)$. This is easy to check for $d=2$ and $d=4$, and then the claim follows by induction using the recursion (4). This yields that

$$
q_{d, n}\left(y_{n}\right)=q_{d-2, n}\left(y_{n}\right) q_{2, n}\left(y_{n}\right)=\left(q_{2, n}\left(y_{n}\right)\right)^{d / 2},
$$

for $d \geq 2$ even. From

$$
y_{n}=1 / n-1 / n^{2}+2 / n^{3}-4 / n^{4}+8 / n^{5}+O\left(1 / n^{6}\right),
$$

we get $q_{2, n}\left(y_{n}\right)=1 / n^{4}+O\left(1 / n^{5}\right)$ and hence

$$
q_{d, n}\left(y_{n}\right)=1 / n^{2 d}+O\left(1 / n^{2 d+1}\right) .
$$

We now show that for sufficiently large $n$ the polynomial $q_{d, n}(x)$ has a root between $y_{n}$ and $w_{d, n}=y_{n}+\frac{2}{n^{2 d+1}}$. We have

$$
q_{d, n}\left(y_{n}\right)=1 / n^{2 d}+O\left(1 / n^{2 d+1}\right)>0 .
$$

By Rolle's theorem, there exists $w_{d, n}^{\prime}$ between $y_{n}$ and $w_{d, n}$ such that

$$
q_{d, n}\left(w_{d, n}\right)=q_{d, n}\left(y_{n}\right)+\left(w_{d, n}-y_{n}\right) q_{d, n}^{\prime}\left(w_{d, n}^{\prime}\right) .
$$


It follows easily by induction that

$$
q_{d, n}(x)=1+(-n+d / 2-2) x+\left((-d / 2+1) n+\left(d^{2}-2 d+8\right) / 8\right) x^{2}+\cdots
$$

Since $y_{n}=1 / n+O\left(1 / n^{2}\right)$, we have $q_{d, n}^{\prime}\left(w_{d, n}^{\prime}\right)=-n-d / 2+O(1 / n)$. Thus, for sufficiently large $n$, we get $q_{d, n}^{\prime}\left(w_{d, n}^{\prime}\right)<-n$. This implies

$$
q_{d, n}\left(w_{d, n}\right)=1 / n^{2 d}+O\left(1 / n^{2 d+1}\right)+\frac{2}{n^{2 d+1}} q_{d, n}^{\prime}\left(w_{d, n}^{\prime}\right)<0 .
$$

Thus, the polynomial $Q_{d, n}(x)=\left(x^{2}-\left(n^{2}+3 n+1\right) x+(n+2)\right) q_{d-2, n}(x)$ has two close roots: $y_{n}$ and $v_{d, n}$, which is between $y_{n}$ and $w_{d-2, n}$. This yields

$$
\operatorname{sep}\left(Q_{d, n}\right) \leq \frac{2}{n^{2 d-3}},
$$

when $n$ is large enough. Since $H\left(Q_{d, n}\right)=O\left(n^{3}\right)$, this gives

$$
e^{*}(d) \geq \frac{2 d-3}{3}
$$

by letting $n$ tend to infinity.

Let now $d$ be odd. Then we define

$$
Q_{d, n}(x)=x\left(x^{2}-\left(n^{2}+3 n+1\right) x+(n+2)\right) q_{d-3, n}(x) .
$$

This polynomial has two close roots: $y_{n}$ and a root lying between $y_{n}$ and $w_{d-3, n}$. Thus we get

$$
\operatorname{sep}\left(Q_{d, n}\right) \leq \frac{2}{n^{2 d-5}}
$$

for $n$ large enough, and

$$
e^{*}(d) \geq \frac{2 d-5}{3}
$$

The proof of Theorem 2 is complete.

\section{Irreducible monic polynomials: Proof of Theo- rem $[3$}

In this section, we use the polynomials $p_{d, n}(x)$ to construct irreducible monic polynomials having two very close roots. Let $F_{k}$ denote the $k$ th Fibonacci number defined by the recursion $F_{0}=0, F_{1}=1$ and $F_{k}=F_{k-1}+F_{k-2}$ for 
$k \geq 2$. Note that Fibonacci numbers appear in the asymptotic expansion of $x_{n}=(n+2) /\left(n^{2}+3 n+1\right)$, namely

$x_{n}=1 / n-1 / n^{2}+2 / n^{3}-5 / n^{4}+13 / n^{5}-34 / n^{6}+\cdots-(-1)^{k} F_{2 k-3} / n^{k}+\cdots$

For $d \geq 0$, we first define monic polynomials $s_{d, n}(x)$ with a root close to $x_{n}$ by

$$
s_{d, n}(x)=(-1)^{d-1}\left(F_{d-1} p_{d, n}(x)-F_{d} x p_{d-1, n}(x)\right),
$$

and then monic polynomials with two close roots by

$$
\begin{aligned}
r_{2 d+1, n}(x) & =x s_{d, n}^{2}(x)+F_{d}^{2} p_{d, n}^{2}(x), \\
r_{2 d, n}(x) & =s_{d, n}^{2}(x)+F_{d-1}^{2} x p_{d-1, n}^{2}(x) .
\end{aligned}
$$

We claim that these polynomials are monic. It suffices to show that this is true for $s_{d, n}(x)$. Since the leading coefficient of $p_{d, n}(x)$ is $F_{d} n+F_{d-2}$, we deduce that the leading coefficient of $s_{d, n}(x)$ is equal to

$$
\begin{gathered}
(-1)^{d-1}\left(F_{d-1}\left(F_{d} n+F_{d-2}\right)-F_{d}\left(F_{d-1} n+F_{d-3}\right)\right) \\
=(-1)^{d-1}\left(F_{d-1} F_{d-2}-F_{d} F_{d-3}\right)=1 .
\end{gathered}
$$

From (2) we get

$$
r_{2 d+1, n}\left(x_{n}\right)=F_{d}^{2} / n^{4 d-1}+O\left(1 / n^{4 d}\right)
$$

and

$$
r_{2 d, n}\left(x_{n}\right)=F_{d-1}^{2} / n^{4 d-3}+O\left(1 / n^{4 d-2}\right)
$$

that is,

$$
r_{d, n}\left(x_{n}\right)=F_{\lfloor(d-1) / 2\rfloor}^{2} / n^{2 d-3}+O\left(1 / n^{2 d-2}\right) .
$$

Observe that the degree of the polynomial $r_{d, n}(x)$ is $d$. We claim that $r_{d, n}(x)$ has two complex conjugate roots $v_{d, n}$ and $\overline{v_{d, n}}$ close to $x_{n}$, more precisely they are equal to

$$
\begin{aligned}
1 / n-1 / n^{2}+2 / n^{3}- & 5 / n^{4}+13 / n^{5}-\ldots+ \\
+ & (-1)^{d} F_{2 d-5} / n^{d-1} \pm i / n^{(2 d-1) / 2}+O\left(1 / n^{d}\right) .
\end{aligned}
$$

Indeed, the polynomials $F_{d}^{2} p_{d, n}^{2}(x)$ and $F_{d-1}^{2} x p_{d-1, n}^{2}(x)$ have double roots $y_{d+1, n}$ and $y_{d, n}$, resp., in the disc $\left|x-x_{n}\right| \leq 1 / n^{2 d+1}$, resp. $\left|x-x_{n}\right| \leq 1 / n^{2 d-1}$. Moreover, we have $F_{d}^{2} p_{d, n}^{2}(x)<x s_{d, n}^{2}(x)$ when $\left|x-x_{n}\right|=1 / n^{2 d+1}$ and $F_{d-1}^{2} x p_{d-1, n}^{2}(x)<s_{d, n}^{2}(x)$ when $\left|x-x_{n}\right|=1 / n^{2 d-1}$. Hence, by Rouché's theorem, the polynomial $r_{d, n}(x)$ has two roots $v$ satisfying $\left|v-x_{n}\right|<1 / n^{d-1}$. 
Using a complex version of Taylor's theorem, by writing the roots $v$ of $r_{d, n}(x)$ close to $x_{n}$ as $v=x_{n}+\beta+\gamma i$ with $\gamma>0$, we get

$$
\begin{aligned}
0 & =r_{d, n}\left(x_{n}+\beta+\gamma i\right) \\
& =r_{d, n}\left(x_{n}\right)+(\beta+\gamma i) r_{d, n}^{\prime}\left(x_{n}\right)+(\beta+\gamma i)^{2} r_{d, n}^{\prime \prime}\left(x_{n}\right) / 2+O\left(1 / n^{3 d-5}\right) .
\end{aligned}
$$

Note that we have

$$
\begin{gathered}
r_{2 d+1, n}^{\prime}\left(x_{n}\right)=\frac{2 F_{d} F_{d+1}}{n^{2 d-1}}+O\left(1 / n^{2 d}\right), \\
r_{2 d, n}^{\prime}\left(x_{n}\right)=\frac{2 F_{d-1} F_{d-2}}{n^{2 d-2}}+O\left(1 / n^{2 d-1}\right),
\end{gathered}
$$

and

$$
r_{d, n}^{\prime \prime}\left(x_{n}\right)=2 F_{\lfloor(d-1) / 2\rfloor}^{2} n^{2}+O(n) .
$$

By considering imaginary parts in the above Taylor's formula we get

$$
\begin{aligned}
\beta & =-\frac{r_{d, n}^{\prime}\left(x_{n}\right)}{r_{d, n}^{\prime \prime}\left(x_{n}\right)}+O\left(\gamma^{-1} r_{d, n}^{\prime \prime}\left(x_{n}\right)^{-1} n^{-3 d+5}\right) \\
& =-\frac{F_{\lfloor(d-1) / 2\rfloor+(-1)^{d}}}{n^{d}}+O\left(\frac{1}{n^{d+1}}\right)+O\left(\frac{1}{\gamma n^{3 d-3}}\right) .
\end{aligned}
$$

Since the distance between the roots $x_{n}+\beta+\gamma i$ and $x_{n}+\beta-\gamma i$ of $r_{d, n}(x)$ is equal to $2 \gamma$ and $H\left(r_{d, n}\right)=O\left(n^{2}\right)$, it follows from Mahler's theorem [11] quoted in the introduction that $\gamma \gg 1 / n^{2 d-2}$. Thus, $\beta=O\left(1 / n^{d-1}\right)$. Moreover, $\beta=O\left(1 / n^{d}\right)$, unless $\gamma \ll 1 / n^{2 d-3}$. Looking at real parts leads to

$$
\begin{aligned}
\frac{F_{\lfloor(d-1) / 2\rfloor}^{2}}{n^{2 d-3}}+\frac{2 \beta F_{\lfloor(d-1) / 2\rfloor} F_{\lfloor(d-1) / 2\rfloor+(-1)^{d}}}{n^{d-2}}+\left(\beta^{2}-\gamma^{2}\right) F_{\lfloor(d-1) / 2\rfloor}^{2} n^{2} & =O\left(\frac{1}{n^{2 d-2}}\right) .
\end{aligned}
$$

The assumption that $\gamma \ll 1 / n^{2 d-3}$ leads to a contradiction, by considering (7) as a quadratic equation in $\beta$. Hence, we have $\beta=O\left(1 / n^{d}\right)$. Now (7) gives $\gamma^{2}=1 / n^{2 d-1}+O\left(1 / n^{2 d}\right)$, i.e., $\gamma=1 / n^{(2 d-1) / 2}+O\left(1 / n^{d}\right)$, as claimed in $(6)$.

Let $R_{d, n}(x)$ be the irreducible factor of $r_{d, n}(x)$ having roots $v_{d, n}$ and $\overline{v_{d, n}}$. Denote by $\delta$ its degree. Note that, since $H\left(r_{d, n}\right)=O\left(n^{2}\right)$, Gelfond's inequality implies that

$$
H\left(R_{d, n}\right)=O\left(n^{2}\right)
$$


Denote by $\operatorname{Res}_{d, n}$ the resultant of the polynomials $R_{d, n}(x)$ and $L_{n}(x)$. Since $\operatorname{Res}_{d, n}$ is a rational integer and $x_{n}$ is not a root of $R_{d, n}(x)$, we have

$$
\left|\operatorname{Res}_{d, n}\right| \geq 1
$$

Furthermore, the definition of the resultant of two polynomials (see e.g. 4, p. 223]) implies that (recall that $R_{d, n}(x)$ is monic)

$$
\left|\operatorname{Res}_{d, n}\right| \leq\left(n^{2}+3 n+1\right)^{\delta} \prod_{t: R_{d, n}(t)=0}\left|t-x_{n}\right| .
$$

Since the product of the absolute values of all the roots of $R_{d, n}(x)$ different from $v_{d, n}$ and $\overline{v_{d, n}}$ is bounded from above by $\sqrt{d+1}$ times the height of $R_{d, n}(x)$ (see [4, Exercise A.1]), we deduce from (6) and (8) that

$$
\left|\operatorname{Res}_{d, n}\right| \ll n^{2 \delta} n^{-2 d+1} n^{2} .
$$

Combined with (9), this gives $2 \delta \geq 2 d-3$, thus

$$
\delta \in\{d, d-1\} .
$$

This implies that either $r_{d, n}(x)$ is irreducible, or it has an integer root (recall that any rational root of a monic polynomial must be a rational integer).

Assume that $r_{d, n}(x)$ is reducible, i.e., that it has an integer root. This integer must divide $r_{d, n}(0)=F_{\lfloor(d-1) / 2 \mid}^{2}$, and, by (5), it is of the form $-\alpha^{2}$ for an integer $\alpha$ dividing $F_{\lfloor(d-1) / 2\rfloor}$. So, for any given degree $d$, there are only finitely many possibilities for the integer root of $r_{d, n}(x)$.

Write $r_{d, n}(x)$ as

$$
r_{d, n}(x)=A_{d}(x) n^{2}+B_{d}(x) n+C_{d}(x) .
$$

By the definition of $r_{d, n}(x)$ and (1), it follows easily by induction that the discriminant $B_{d}(x)^{2}-4 A_{d}(x) C_{d}(x)$ of $r_{d, n}(x)$ with respect to $n$ is equal to $-4 F_{\lfloor(d-1) / 2\rfloor}^{4} x^{2 d-1}$. In particular, this discriminant is nonzero at $x=-\alpha^{2}$, and thus at least one of the numbers $A_{d}\left(-\alpha^{2}\right), B_{d}\left(-\alpha^{2}\right), C_{d}\left(-\alpha^{2}\right)$ is nonzero. This shows that $r_{d, n}(x)$ is irreducible over $\mathbb{Z}[n, x]$.

Furthermore, for given $\alpha$ there are exactly two (rational) values of $n$ such that $-\alpha^{2}$ is a root of $r_{d, n}(x)$. Hence, for sufficiently large positive integer $n$, the polynomial $r_{d, n}(x)$ has no integer roots, and therefore it is irreducible over $\mathbb{Z}[x]$.

Since

$$
\operatorname{sep}\left(r_{d, n}\right)=O\left(n^{-(d-1 / 2)}\right)
$$


we obtain

$$
e_{\mathrm{irr}}^{*}(d) \geq \frac{2 d-1}{4}
$$

which proves Theorem 3 .

\section{Clusters of roots}

Let $d \geq 2$ be an integer. Mahler's upper bound $e(d) \leq d-1$ quoted in the Introduction is a particular case of the lower bound

$$
\prod_{1 \leq i<j \leq k}\left|\alpha_{i}-\alpha_{j}\right| \gg H(P)^{-d+1}
$$

valid for any integer polynomial $P(x)$ of degree $d$ having at least $k \geq 2$ distinct roots $\alpha_{1}, \ldots, \alpha_{k}$, established in [11].

In [7] the authors extended the definition of $e(d)$ to clusters of at least 3 roots. Let $k$ and $d$ be integers with $2 \leq k \leq d$. We denote by $E(d, k)$, respectively $E_{\mathrm{irr}}(d, k)$, the infimum of the real numbers $\delta$ for which

$$
\prod_{1 \leq i<j \leq k}\left|\alpha_{i}-\alpha_{j}\right| \geq H(P)^{-\delta}
$$

holds for every integer polynomial $P(x)$, respectively, irreducible integer polynomial $P(x)$, of degree $d$ and sufficiently large height, with distinct roots $\alpha_{1}, \ldots, \alpha_{d}$. We further use the notation $E^{*}(d, k)$, respectively $E_{\mathrm{irr}}^{*}(d, k)$, when we restrict our attention to monic integer polynomials, respectively monic integer irreducible polynomials.

We deduce from (10) that the quantities $E(d, k)$ and $E^{*}(d, k)$ are all bounded from above by $d-1$. Regarding lower bounds, it is proved in [6, 7] that, for any integer $d \geq 4$ and any integer $k \geq 2$ that divides $d$, we have

$$
E(d, k) \geq \frac{k-1}{k} d \quad \text { and } \quad E^{*}(d, k) \geq \frac{k-1}{k} d-\frac{k-1}{2} .
$$

In fact, the results from [6, 7] show that these bounds are valid also for $E_{\text {irr }}(d, k)$ and $E_{\text {irr }}^{*}(d, k)$, respectively. The constructions presented in the Sections 2 and 3 allow us to strengthen the estimates (11).

Theorem 4 For every integer $k \geq 3$, there exist rational numbers $c_{1}(k)$ and $c_{2}(k)$ such that, for every integer $d \geq k$, we have

$$
E(d, k) \geq \frac{k}{k+1} d-c_{1}(k)
$$


and

$$
E^{*}(d, k) \geq \frac{k}{k+1} d-c_{2}(k) .
$$

In particular, the exponent $-d+1$ in (10) cannot be replaced by $-\alpha_{k} d$, for a real number $\alpha_{k}$ less than $k /(k+1)$, even if the polynomial $P(x)$ in (10) is assumed to be monic.

Proof. Let $\delta \geq 2$ and $h \geq 0$ be integers. The polynomial

$$
\tilde{P}_{\delta, h, n}(x):=P_{\delta, n}(x) \times p_{\delta, n}(x) \times \cdots \times p_{\delta+h, n}(x)
$$

has degree $(h+2) \delta+h(h+1) / 2$, height $\ll n^{h+4}$, and it has a cluster of $h+3$ roots close to each other. Setting $k=h+3$, a short calculation gives (12), since one can multiply $\tilde{P}_{\delta, h, n}(x)$ by a suitable power of the monomial $x$. To get (13), it suffices, for $\delta$ even, to consider the polynomial

$$
\tilde{Q}_{\delta, h, n}(x):=Q_{\delta, n}(x) \times q_{\delta, n}(x) \times \cdots \times q_{\delta+2 h, n}(x),
$$

multiplied by a suitable power of the monomial $x$. We omit the details.

\section{References}

[1] V. Beresnevich, V. Bernik, and F. Götze, The distribution of close conjugate algebraic numbers, Compositio Math. 146 (2010), 1165-1179.

[2] E. Bombieri, On the Thue-Siegel-Dyson theorem, Acta Math. 148 (1982), 255-296.

[3] Y. Bugeaud, Mahler's classification of numbers compared with Koksma's, Acta Arith. 110 (2003), 89-105.

[4] Y. Bugeaud, Approximation by algebraic numbers. Cambridge Tracts in Mathematics, Cambridge, 2004.

[5] Y. Bugeaud and A. Dujella, Root separation for irreducible integer polynomials, Bull. Lond. Math. Soc. 43 (2011), 1239-1244.

[6] Y. Bugeaud and M. Mignotte, On the distance between roots of integer polynomials, Proc. Edinburgh Math. Soc. 47 (2004), 553-556.

[7] Y. Bugeaud and M. Mignotte, Polynomial root separation, Intern. J. Number Theory 6 (2010), 587-602. 
[8] A. Dubickas, Polynomial root separation in terms of the Remak height, Turkish Journal of Mathematics 37 (2013), 747-761.

[9] A. Dujella and T. Pejković, Root separation for reducible monic quartics, Rend. Semin. Mat. Univ. Padova 126 (2011), 63-72.

[10] J.-H. Evertse, Distances between the conjugates of an algebraic number, Publ. Math. Debrecen 65 (2004), 323-340.

[11] K. Mahler, An inequality for the discriminant of a polynomial, Michigan Math. J. 11 (1964), 257-262.

[12] A. Schönhage, Polynomial root separation examples, J. Symbolic Comput. 41 (2006), 1080-1090.

Yann Bugeaud

Université de Strasbourg

Département de Mathématiques

7, rue René Descartes

67084 Strasbourg, France

E-mail address: bugeaud@math.unistra.fr

Andrej Dujella

Department of Mathematics

University of Zagreb

Bijenička cesta 30

10000 Zagreb, Croatia

E-mail address: duje@math.hr 